\title{
ANÁLISE DAS FORMAÇÕES COLETIVAS OFERTADAS AOS TRABALHADORES DE UM HOSPITAL PÚBLICO: O CASO DO GRUPO HOSPITALAR CONCEIÇÃO
}

\author{
ANALYSIS OF COLLECTIVE TRAINING OFFERED TO WORKERS OF A \\ PUBLIC HOSPITAL: THE CASE OF GRUPO HOSPITALAR CONCEIÇÃO
}

\section{Fabricio Felipe Quadros}

Mestre em Reabilitação e Inclusão. Técnico em Educação na Gestão do Trabalho do Grupo Hospital Conceição.

E-mail: qfabricio@ghc.com.br

\section{Lisiane Boer Possa}

Doutora em Sociologia. Professora na Saúde Coletiva da Universidade Federal do Rio Grande do Sul. E-mail: lisianepossa@gmail.com

\section{Resumo}

Este estudo buscou analisar e descrever atividades de formação coletivas ofertadas aos trabalhadores do GHC, verificando quais tecnologias do cuidado se associam à formação de 2013. Tem-se como pressuposto que as atividades de formação refletem o modelo tecnoassistencial da instituição. Buscouse caracterizar oferta de atividades considerando: responsáveis que as desenvolvem, analisar a oferta para as áreas meio e assistencial, e distinguir entre aquelas oferecidas institucionalmente e pelas equipes; categorias profissionais atuantes no GHC que realizaram formação; tipologias das atividades formativas ofertadas e as temáticas ofertadas e sua relação com as tecnologias do cuidado, modos de produção e modelagens tecnoassistenciais em saúde. Este estudo caracterizou-se como Estudo de Caso descritivo de análise documental com abordagem quanti-qualitativa. ${ }^{1}$ Observamos que as equipes foram as maiores responsáveis pela formação, ofertando atividades voltadas para o segmento assistência/fim. Já as institucionais foram atividades caracterizadas pela participação significativa do segmento apoio/meio. Percebeuse, a tentativa de proporcionar atividades integrativas entre esses segmentos. Verficouse que os trabalhadores assistenciais foram os maiores beneficiados e que trabalhadores de alguns cargos apresentam poucos concluintes considerando sua representação no GHC. As 
atividades mais realizadas são: Treinamento ou Capacitação, possuindo características de transmissão de conhecimento, sendo antagonista às características da educação permanente, refletindo nos resultados das tecnologias do cuidado. Os resultados tecnologias leve/duras, representaram o maior quantitativo das formações, seguida pelas tecnologias duras, o que reflete o modelo tecnoassistencial hegemônico na instituição. Espera-se que este estudo possa servir de reflexão para contribuir para qualificar os processos de formação coletivos realizados no GHC.

Palavras-chave: Educação em Saúde, Educação Permanente, Tecnologias do Cuidado em Saúde, Modelos Tecnoassistenciais.

\section{Abstract}

This study aimed to analyze and describe collective training activities offered to workers of $\mathrm{GHC}$, checking which technologies of healthcare are associated with the formation of 2013. It has been assumptioned that the training activities reflect the technical healthcare model of the institution. We sought to characterize supply activities considering: responsibles for its development, analyze the offer to the essencial and assistance areas, and to distinguish between those offered by the institution and those by the teams; professional categories active in GHC and trained ones; typologies of the training activities offered and theme issues offered and their relationship with care technology, production modes and modeling technical healthcare in health. This study characterized as a descriptive study of documentary analysis case with quantitative and qualitative approach. ${ }^{1}$ Were observed that teams are most responsible for training, offering activities aimed for the assistance/end segment. On the other hand, institutional activities were characterized for significant participation on the support/ essential segment. It was noticed an attempt to provide activities of integrative processes between these segments. It was pointed that the assistance workers were the most beneficiaries and that workers from some posts had few graduates considering their roles at GHC. The most activities carried out are: training or capacity building, possessing characteristics of knowledge transmission, being antagonistic of continued education, reflecting at healthcare technologies results. Results technologies lightweight/ hard, represented the major quantitative of formation, followed by hard technologies, that reflects that the technical healthcare hegemonic model in the institution. It is expected that this study can serve as a reflection to contribute to qualify the collective training processes done at GHC.

Keywords: Health care education, Permanent education, Technologies of health care at health, Technical health care models.

\section{Introdução}

A pesquisa realizada descreve e analisa as atividades coletivas de formação, ofertadas para os trabalhadores do Grupo Hospitalar Conceição - GHC, utilizando como recorte o ano de 2013. O GHC é uma instituição de saúde, vinculada ao Ministério da Saúde, que está situada em Porto Alegre/ RS. Buscou-se analisar e descrever as atividades de formação e a caracterização dos trabalhadores formados, nas ofertas realizadas pela instituição. ${ }^{2}$ 
Tem-se como pressuposto que as atividades formativas realizadas refletem a modelagem tecnoassistencial presente na instituição, portanto, a análise sobre a formação ofertada contribuiria para embasar discussões sobre as políticas de formação e reorganização do modelo de atenção à saúde, propostas pelo Sistema Único de Saúde - SUS e assumidas como compromisso na instituição. ${ }^{3}$

O GHC é uma instituição 100\% Sistema Único de Saúde (SUS), com cerca de 8.500 trabalhadores. Tem como objetivo explicitado, nas suas diretrizes, o atendimento das reais necessidades da população, ou seja, a saúde é tratada como um direito constitucional das pessoas. O GHC organiza suas normativas baseadas nos princípios do Sistema Único de Saúde (SUS) e nas políticas do Ministério da Saúde, com orientação estratégica do Governo Federal, sendo que, nos últimos anos, propôs políticas institucionais de democratização, de qualificação dos trabalhadores e de gestão do trabalho em sintonia com essas diretrizes. ${ }^{4}$

A política de qualificação dos trabalhadores inclui a realização institucional de atividades educativas, desenvolvidas pelas áreas responsáveis pela formação no GHC: Gestão do Trabalho, Educação e Desenvolvimento (GTED), Escola GHC e Gerências, bem como aquelas desenvolvidas pelas próprias equipes em que estão os trabalhadores. ${ }^{2,5}$

\section{A formação dos trabalhadores da saúde - uma política em construção}

No ano de 2006, a Política Nacional de Educação Permanente em Saúde, é lançada no intuito de nortear as responsabilidades das três esferas de gestão do SUS, relativas à gestão da Educação na Saúde. $6,7,8,9$
Como pressuposto, a Educação Permanente na saúde deve implicar os sujeitos com seu próprio processo de trabalho, colocando, em perspectiva, o desafio de pensar novas possibilidades pedagógicas que possibilitem a formação de sujeitos autônomos, comprometidos com o conceito de qualidade de vida, no âmbito individual e coletivo, tendo, como centro do processo pedagógico, a implicação do trabalhador no momento exato do ato do trabalho, produzindo cuidado em saúde. ${ }^{10,11,12}$

Pode-se associar a Educação Permanente em saúde a uma pedagogia em ato e, conseqüentemente, a um trabalho vivo. O exercício da saúde é amplamente dependente do trabalho vivo em ato, onde os trabalhadores podem colocar todo conhecimento de que dispõem como opções tecnológicas para a produção de modos de proceder eficaz, a serviço do usuário e de seu problema. ${ }^{13,14,15}$

No processo de produção do cuidado, as três tecnologias (duras, leve/ duras e leves) estão presentes. Conforme o modelo tecnoassistencial adotado, há diferentes modos destas se configurarem. No modelo médico-hegemônico, ocorre um predomínio das tecnologias duras sobre as outras, inclusive comandando, ou mesmo, esvaziando a presença das outras tecnologias nos seus atos produtivos. Já, nos modelos centrados no mundo das necessidades de vida, dos usuários, há que se deslocar o centro tecnológico para o mundo das tecnologias leves, relacionais. ${ }^{13,14,15}$

Entre produção de cuidado e produção pedagógica, quando se refere à Educação Permanente na saúde, significa que um está no outro, isso é, o trabalho está na pedagogia e esta na atividade laboral, e eles só têm a potência de produzir Educação Permanente quando há essa transversalidade. ${ }^{16}$

Quando a organização técnica do trabalho for favorável ao trabalho morto, reflete um processo de trabalho dependente 
das tecnologias duras e leves-duras, voltado à produção de procedimentos, baseado em uma formação tecnicista ou tradicional de operacionalização de processos. Caso contrário, quando houver predominância do trabalho vivo em ato, haverá uma produção do cuidado, centrado nas tecnologias leves, em que o projeto é centrado no usuário, sendo o trabalhador autônomo para decidir a melhor forma de utilizar os processos e fluxos adequadamente à necessidade de saúde do usuário ou da comunidade. ${ }^{17}$

Nesse sentido o objetivo geral desta pesquisa foi descrevere analisar as atividades coletivas de formação dos trabalhadores do GHC, desenvolvidas na instituição, no ano de 2013. Caracterizando a oferta de atividades considerando: 1) os responsáveis que as desenvolvem, analisando a oferta para as áreas meio e assistencial ${ }^{1} \mathrm{e}$ a distinção entre aquelas oferecidas institucionalmente e pelas equipes; 2 ) as categorias profissionais que atuam no GHC e realizaram formação; 3) as tipologias das atividades formativas ofertadas e; 4) as temáticas que foram ofertadas e sua relação com as diferentes tecnologias do cuidado (tecnologias duras, leve-duras e leves), os modos de produção e as modelagens tecnoassistenciais em saúde.

\footnotetext{
Áreas meio são aquelas relativas às atividades que se destinam como um apoio à área assistencial, que é diretamente relacionada com o cuidado aos usuários do serviço, a área assistencial também é denominada área fim nos serviços de saúde.
}

\section{Métodos}

Este estudo caracterizou-se como Estudo de Caso descritivo de análise documental com abordagem quantiqualitativai.

Para análise das atividades de formação coletivas, foi realizado um recorte do ano de 2013. Este período foi escolhido e utilizado por apresentar um banco de dados mais confiável em comparação aos anos anteriores. Também, a tentativa é aproximar essas atividades à proposta de educação permanente, só sendo possível analisando o recorte das atividades coletivas, com aproximação às características da educação permanente.

Portanto, as atividades de iniciativa pessoal ou de nível acadêmico não fizeram parte da pesquisa, e sim, as atividades realizadas coletivamente pela instituição e suas equipes, para os trabalhadores do GHC.

Nesse sentido para realizar a revisão e sistematização dos conceitos de educação em saúde, modos de produção, tecnologias de cuidado e modelos tecnoassistenciais, elementos centrais neste trabalho, foi realizada pesquisa bibliográfica utilizando-se desta terminologia. Os demais objetivos propostos foram respondidos através da análise de dados, disponível no sistema informatizado da instituição (GHC-Sistemas). ${ }^{2}$ A análise documental, disponível no Núcleo de Atividades de Formação (NAF), que é um núcleo da GTED. ${ }^{19,20}$ Esse núcleo possui suas informações sistematizadas através de planilhas e relatórios informatizados, compondo uma base de dados secundária que sistematiza o conjunto das atividades formativas realizadas no $\mathrm{GHC} .{ }^{19,20}$ 


\section{Resultados e discussão}

Tabela 1: Atividades de formação realizadas pela instituição e pelas equipes no GHC no ano de 2013.

\begin{tabular}{l|c|c}
\hline \multicolumn{1}{c|}{ Número de Atividades Realizadas } & Total & Percentual \\
\hline Institucional & 381 & $16 \%$ \\
\hline Equipe & 2.043 & $84 \%$ \\
\hline Total & 2.424 & $100 \%$ \\
\hline
\end{tabular}

Fonte: Fonte: NAF (Núcleo Atividade de Formação) GTED do GHC.

É possível identificar que, ao todo foram, desenvolvidas 2.424 atividades no ano de 2013. A maior parte delas foram realizadas pelas equipes, $84 \%$, enquanto o percentual de atividades realizadas pela instituição, seja por meio da Gestão do Trabalho, Educação e Desenvolvimento ou pela Escola GHC, foi de $16 \%$, do total das atividades realizadas. Esses dados sinalizam que as equipes são as principais protagonistas dos processos formativos coletivos no GHC, podendo favorecer a Educação Permanente, uma vez que os gestores e os trabalhadores da ponta têm autonomia para, no cotidiano, definirem as temáticas e os métodos de formação que desenvolverão.

A Tabela 2 apresenta os dados sobre o número e o percentual de atividades realizadas, por segmento, pela instituição e pelas equipes.

Tabela 2: Atividades de formação realizadas, pela instituição e pelas equipes, em 2013, considerando para quais segmentos se destinam.

\begin{tabular}{l|c|c|c|c|c|c}
\hline Número de Atividades Realizadas & Institucional & $\%$ & Equipe & $\%$ & Total & $\%$ \\
\hline Assistência/Fim & 107 & $27 \%$ & 1999 & $98 \%$ & 2106 & $87 \%$ \\
\hline Apoio/Meio & 20 & $5 \%$ & 16 & $1 \%$ & 36 & $1 \%$ \\
\hline Ambos & 260 & $68 \%$ & 28 & $1 \%$ & 288 & $12 \%$ \\
\hline Total & 381 & $100 \%$ & 2043 & $100 \%$ & 2424 & $100 \%$ \\
\hline
\end{tabular}

Fonte: NAF (Núcleo Atividade de Formação) GTED do GHC.

Pode-se observar que, das $68 \%$ das atividades ofertadas pela instituição, foram organizadas para formação multiprofissional, contemplando tanto trabalhadores do segmento Assistência/Fim quanto trabalhadores do segmento Apoio/Meio. Isso aponta a idéia do trabalho sob a lógica da Educação Permanente, uma vez que uma característica desse processo é a participação envolvendo os diversos atores e contemplando a lógica multiprofissional. 


\section{Artigo de Original}

Para as equipes, que são as maiores protagonistas dos processos formativos, pode-se destacar o expressivo volume de atividades de formação oferecidas para os trabalhadores do segmento Assistência/Fim, representando $98 \%$ do total de atividades realizadas pelas equipes. Quando analisamos os percentuais, observa-se que, $87 \%$ atividades beneficiaram os trabalhadores do segmento Assistência/Fim, somando-se $12 \%$ atividades de formação ofertadas para ambos os segmentos, $99 \%$ de atividades de formação com acesso aos trabalhadores do segmento Assistência/Fim.

Mesmo observando-se uma tentativa da instituição de organizar atividades multiprofissionais englobando trabalhadores do segmento Apoio/Meio, ainda tendem a desenvolver processos que podem ser multiprofissionais, porém possui seu foco em cargos assistenciais, muitas vezes, excluindo trabalhadores da própria equipe que tenham cargos do segmento Apoio/Meio.

A Educação Permanente pressupõe participação e, ao se observar segmentos afastados dessa perspectiva, demonstra-se que desenvolver um processo de formação baseado nesse modelo ainda pode ser considerado um desafio em alguns espaços.

Além disso, é possível identificar, a partir disso, uma desproporcionalidade no acesso às atividades de formação coletivas entre os dois segmentos.

A Tabela 3 apresenta o número de trabalhadores do GHC e dos trabalhadores concluintes de cursos por cargo e a média de atividades formativas por trabalhador no GHC em 2013. Essa informação tem como objetivo verificar a relação entre os trabalhadores existentes e os trabalhadores concluintes de atividades formativas para cada cargo, oportunizando a análise da equidade da distribuição da formação considerando o número de trabalhadores de cada cargo que atua no GHC no mesmo período.

Tabela 3: Número de trabalhadores do GHC e dos concluintes de cursos por cargo e a média de atividades formativas por trabalhador por cargo em 2013.

(continua)

\begin{tabular}{|c|c|c|c|}
\hline Cargos & $\begin{array}{c}\mathrm{N} \text { de } \\
\text { Trabalhadores por } \\
\text { Cargos no GHC }\end{array}$ & $\begin{array}{l}\text { Número de } \\
\text { concluintes } \\
\text { de Formações } \\
\text { ofertados }\end{array}$ & $\begin{array}{l}\text { Média de atividades } \\
\text { formativas por } \\
\text { trabalhador }\end{array}$ \\
\hline *Administrativo Superior & 132 & 449 & 3,4 \\
\hline **Administrativo Médio & 938 & 3.921 & 4,2 \\
\hline $\begin{array}{l}\text { ***Cozinheiro/Despenseiro/Nutrição } \\
\text { nível Médio e Elementar }\end{array}$ & 586 & 6.449 & 11 \\
\hline ****Enfermagem Médio/Elementar & 3.367 & 26.902 & 8 \\
\hline Assistente Social/TO & 76 & 911 & 12 \\
\hline Auxiliar de Almoxarifado & 19 & 54 & 2,8 \\
\hline Auxiliar de Farmácia & 109 & 1.195 & 11 \\
\hline Agente Saúde Comunitária & 8 & 27 & 3,3 \\
\hline Fisioterapeuta/Auxiliar de Fisiatria & 82 & 655 & 7,9 \\
\hline
\end{tabular}


Artigo de Original

(conclusão)

\begin{tabular}{|c|c|c|c|}
\hline $\begin{array}{l}* * * * * \text { Laboratório Médio e } \\
\text { Elementar }\end{array}$ & 63 & 923 & 14,6 \\
\hline Técnico/Auxiliar de Radiologia & 143 & 613 & 4,2 \\
\hline $\begin{array}{l}* * * * * * \text { Manutenção Médio e } \\
\text { Elementar }\end{array}$ & 172 & 863 & 5 \\
\hline Auxiliar Geral & 309 & 1.265 & 4 \\
\hline Biólogo/Químico & 3 & 26 & 8,6 \\
\hline Costureiro & 3 & 11 & 3,6 \\
\hline Farmacêutico/Bioquímico & 137 & 1.291 & 9,4 \\
\hline Fonoaudiólogo & 18 & 81 & 4,5 \\
\hline Instrumentador Cirúrgico & 4 & 10 & 2,5 \\
\hline Médico & 1.159 & 6.764 & 5,8 \\
\hline Motorista & 20 & 4 & 0,2 \\
\hline Nutricionista & 82 & 1.134 & 13,8 \\
\hline Odontólogo & 65 & 496 & 7,6 \\
\hline Programador & 8 & 14 & 1,7 \\
\hline Psicólogo & 48 & 613 & 12,7 \\
\hline Enfermeiro & 754 & 10.180 & 13,5 \\
\hline Segurança Trabalho/Vigia & 145 & 447 & 3 \\
\hline $\begin{array}{l}\text { Técnico em Saúde Bucal/Prótese } \\
\text { dentária }\end{array}$ & 28 & 364 & 13 \\
\hline Telefonista & 22 & 73 & 3,3 \\
\hline Total & 8.500 & 65.462 & 7,70 \\
\hline
\end{tabular}

*Administrador, Advogado, Bibliotecário, Técnico em Educação, Analista de Sistemas, Engenheiro, Contador, Arquiteto, Analista de Suporte, Auditor.

**Auxiliar Administrativo, Auxiliar Técnico Administrativo, Técnico Administrativo, Supervisor Administrativo, Supervisor Especializado, Técnico Especializado.

***Cozinheiro, Despenseiro, Técnico de Nutrição, Atendente de Nutrição, Auxiliar de Nutrição.

****Técnico de Enfermagem, Gessista, Atendente de Enfermagem, Auxiliar de Enfermagem.

*****Técnico em Histopatologia, Auxiliar de Laboratório, Auxiliar de Limpeza Esterilização de Laboratório, Coletador de Laboratório, Auxiliar Técnico de Banco de Sangue, Auxiliar Técnico de Gasometria.

******Técnico de Manutenção, Técnico em Eletrônica, Auxiliar de Manutenção, Especialista em Manutenção Hospitalar. 
Quando observamos o número de trabalhadores do cargo em relação ao total de trabalhadores do GHC, é possível observar que os cargos classificados como: Enfermagem Médio/Elementar representam 39\%, Médicos, 14\%, Administrativos Médios, 11\% e Enfermeiros, $9 \%$. Somando esse quantitativo, obtém-se um percentual $73 \%$ do total de trabalhadores do GHC, esse resultado demonstra que os trabalhadores com cargos assistenciais concentram o grande quantitativo de trabalhadores do GHC.

Observando o número de concluintes de formações ofertadas do cargo em relação ao total de concluintes de formações do GHC, os cargos de Enfermagem Médio/Elementar representam $41 \%$ concluintes, seguidos pelos cargos Enfermeiros, com 16\% concluintes, Médicos, 10\% concluintes, e Cozinheiro/Despenseiro/Nutrição nível Médio e Elementar, com $10 \%$ concluintes. Tais resultados somados representam $77 \%$ do total de concluintes das atividades formativas coletivas ofertadas em 2013. Esses dados reforçam que há proporcionalidade entre os cargos existentes e os concluintes das atividades formativas, uma vez que o maior volume de trabalhadores com cargos assistenciais também representaram o maior número de concluintes das atividades de formação coletivas realizadas pelo GHC em 2013.

Cabe destacar que a única categoria que teve menor número de concluintes do que o número de trabalhadores existentes foi a de motorista 0,2 atividades por trabalhador. Os trabalhadores dos demais cargos tiveram mais atividades formativas que o número de trabalhadores, pressupondo que, ao menos, uma atividade formativa foi realizada para cada trabalhador do GHC no período, sendo que os trabalhadores do laboratório, médio e elementar tiveram o maior número de atividades formativas 15 por trabalhador.

Considerando esses dados, é possível constatar que as atividades formativas envolveram todos os profissionais da assistência. Há que se considerar, também, para aprofundar essa análise, o quanto tais formações eram multiprofissionais ou uniprofissionais, o que não foi possível verificar neste trabalho a partir dos dados existentes.

A Tabela 4 apresenta os dados de número total e percentual de atividades por tipos de formação realizadas pela instituição e pelas equipes em 2013.

Tabela 4: Número total e percentual dos tipos de formação realizadas pela instituição e pelas equipes em 2013.

(continua)

\begin{tabular}{l|c|c|c|c|c|c}
\hline \multicolumn{1}{c|}{ Tipo de Formação } & Institucional & $\%$ & Equipe & $\%$ & Total & $\%$ \\
\hline $\begin{array}{l}\text { Treinamento ou } \\
\text { Capacitação }\end{array}$ & 140 & $36,7 \%$ & 988 & $48,4 \%$ & 1128 & $46,5 \%$ \\
\hline Aulas e Palestras & 67 & $17,6 \%$ & 681 & $33,3 \%$ & 748 & $30,8 \%$ \\
\hline Oficina & 16 & $4,2 \%$ & 27 & $1,3 \%$ & 43 & $1,8 \%$ \\
\hline Seminário & 9 & $2,3 \%$ & 124 & $6 \%$ & 133 & $5,5 \%$ \\
\hline Encontro & 7 & $1,8 \%$ & 37 & $1,8 \%$ & 44 & $1,8 \%$ \\
\hline Curso & 127 & $33,3 \%$ & 41 & $2 \%$ & 168 & $6,9 \%$ \\
\hline
\end{tabular}


Artigo de Original

(conclusão)

\begin{tabular}{l|c|c|c|c|c|c}
\hline $\begin{array}{l}\text { Grupo de Estudo/ } \\
\text { Estudo de caso }\end{array}$ & 0 & $0 \%$ & 76 & $3,7 \%$ & 76 & $3 \%$ \\
\hline Mesa Redonda & 3 & $0,5 \%$ & 2 & $0,1 \%$ & 5 & $0,2 \%$ \\
\hline Atualização & 1 & $0,3 \%$ & 29 & $1,4 \%$ & 30 & $1,2 \%$ \\
\hline Colóquio & 0 & $0 \%$ & 1 & $0,1 \%$ & 1 & $0,1 \%$ \\
\hline Conferência/ & 0 & $0 \%$ & 13 & $0,6 \%$ & 13 & $0,5 \%$ \\
\hline Teleconferência & 7 & $1,8 \%$ & 1 & $0,1 \%$ & 8 & $0,3 \%$ \\
\hline Congresso & 1 & $0,3 \%$ & 0 & $0 \%$ & 1 & $0,1 \%$ \\
\hline Córumvenção & 0 & $0 \%$ & 6 & $0,3 \%$ & 6 & $0,2 \%$ \\
\hline Jornada & 3 & $0,9 \%$ & 4 & $0,2 \%$ & 7 & $0,3 \%$ \\
\hline Mostra & 1 & $0,3 \%$ & 1 & $0,1 \%$ & 2 & $0,1 \%$ \\
\hline Plenária & 0 & $0 \%$ & 1 & $0,1 \%$ & 1 & $0,1 \%$ \\
\hline Programa & 0 & $0 \%$ & 1 & $0,1 \%$ & 1 & $0,1 \%$ \\
\hline Projeto & 0 & $0 \%$ & 2 & $0,1 \%$ & 2 & $0,1 \%$ \\
\hline Simpósio & 0 & $0 \%$ & 2 & $0,1 \%$ & 2 & $0,1 \%$ \\
\hline Videoaulas/ & 0 & $0 \%$ & 3 & $0,1 \%$ & 3 & $0,1 \%$ \\
\hline videoconferências & 0 & $0 \%$ & 1 & $0,1 \%$ & 1 & $0,1 \%$ \\
\hline Wisita Técnica & 0 & $100 \%$ & 2043 & $100 \%$ & 2424 & $100 \%$ \\
\hline Total & 0 & $0,1 \%$ & 2 & $0,1 \%$ \\
\hline
\end{tabular}

Fonte: NAF (Núcleo Atividade de Formação) GTED do GHC.

No ano de 2013, as atividades classificadas como "treinamento ou Capacitação", somaram $46 \%$ do total, as classificadas como "Aulas e Palestras", $31 \%$ do total, representando $77 \%$ do total de atividades realizadas para os trabalhadores do GHC.

É possível observar, através dos dados, uma tendência maior de formações com características duras ou leve/duras, do ponto de vista das tecnologias do trabalho em saúde, analisando as características das atividades classificadas como "treinamento ou capacitação" e "aulas e palestras". Essas atividades têm característica de transmissão de conhecimento, em uma perspectiva de atividade expositiva, onde o facilitador detém o conhecimento e o transmite ao ouvinte (trabalhador). Esse modelo de formação dificilmente abre espaços para a construção coletiva, para o questionamento e para a reflexão do processo de trabalho 
em saúde. Nesses modelos, o trabalhador não ocupa um espaço de protagonismo nos seus processos, e sim, de simples operador. Tais tipos de formação apresentam características mais aproximadas do modelo tecnoassistencial hegemônico, centrando nos procedimentos e nas especialidades, e cognitivista, do ponto de vista da proposta educativa.

A Tabela 5 apresenta os dados de número total e percentual de formação realizada pela instituição e pelas equipes em 2013, considerando a tipologia das tecnologias do trabalho em saúde envolvidas.

Tabela 5: Número total e percentual de formação realizadas pela instituição e pelas equipes, em 2013, considerando a tipologia das tecnologias do trabalho em saúde.

\begin{tabular}{l|c|c|c|c|c|c}
\hline $\begin{array}{l}\text { Tecnologias do Trabalho } \\
\text { em Saúde }\end{array}$ & Institucional & $\%$ & Equipe & $\%$ & Total & \% Total \\
\hline Leve & 35 & 10 & 348 & 17 & 383 & 16 \\
\hline Leve/Dura & 299 & 78 & 1.303 & 64 & 1.602 & 66 \\
\hline Dura & 47 & 12 & 392 & 19 & 439 & 18 \\
\hline Total & 381 & 100 & 2.043 & 100 & 2.424 & 100 \\
\hline
\end{tabular}

Fonte: dados da pesquisa.

A classificação das atividades conforme a tipologia das tecnologias em saúde foi construída a partir da análise realizada, considerando-se as informações do tipo de formação e o título das atividades. Essa caracterização foi possível considerando as informações disponíveis, mas acredita-se que oportunizaram uma classificação aproximada das formações em relação à tecnologia do trabalho em saúde para a qual se destinavam. Tanto nas atividades formativas institucionais como nas das equipes, observa-se um quantitativo e um percentual mais elevado de tecnologias Leve/dura, seguido pelas tecnologias duras e pelas tecnologias leves.

Somando todas as atividades realizadas coletivamente pelo GHC, em 2013, observa-se um percentual de $66 \%$ de atividades que se aproximam das características de leve/duras, $18 \%$ de duras e $16 \%$ de leves.

Podemos associar esses resultados, articulados aos tipos de formação realizados no GHC, ao modelo tecnoassistencial prevalente no Grupo, que é mais aproximado de um processo centrado no procedimento e no profissional médico, distanciado dos princípios e das diretrizes do SUS, que pressupõem que a centralidade dos processos de cuidado terapêutico esteja no usuário e na construção desse cuidado através do trabalho vivo, baseado na interação e na troca que se dá nesta relação.

\section{Considerações Finais}

Neste estudo, foram analisadas as ofertas das atividades coletivas de formação realizadas no GHC, em 2013. Verificamos também os responsáveis pelas formações, o acesso às atividades pelos segmentos apoio/meio e assistência/fim e os quantitativos e percentuais de atividades realizadas pela instituição e pelas equipes. Por fim, foram observadas as categorias profissionais mais beneficiadas, os tipos de atividade ou a característica das atividades realizadas, bem 
como a relação dos tipos de formação e os temas das formações com as tecnologias do cuidado em saúde, os modos de produção e o modelo tecnoassistencial.

Pode-se constatar, ao final desta pesquisa, que os pensadores da educação em saúde, citados no trabalho, associam a Educação Permanente em saúde e suas características as tecnologias do cuidado, com aproximação mais evidente às tecnologias leves: caracterizadas pelo trabalho integrado e humanizado realizado no ato do cuidado, baseado na construção compartilhada do projeto terapêutico, centrado no usuário, dependente da relação que é estabelecida entre trabalhador de saúde e usuário.

Conseqüentemente, o modelo tecnoassistencial que mais se aproxima dessa proposta é o modelo contrahegemônico, centrado no usuário, que valoriza todas as formações e todos os olhares, em busca de um objetivo comum, tratar, de forma multiprofissional, com integralidade e humanização, a necessidade do usuário, respeitando sua individualidade de forma equânime.

A Educação Permanente parte do pressuposto que todos podem contribuir, que a terapêutica não deve ser centrada em apenas um profissional, subaproveitando os demais conhecimentos, parte de um processo reflexivo que aflora do processo de trabalho e que, coletivamente, deve ser analisado, deve ter diagnóstico, deve ser discutido e, por fim, deve resultar ações de qualificação e superação das dificuldades observadas, baseadas no modelo que se quer constituir.

Lembra-se que este estudo realizou um recorte, analisando apenas as atividades de formação coletivas, realizadas no ano de 2013, e que existem outras formas de acesso para formação no GHC. Pode-se perceber que as equipes foram as maiores responsáveis por ofertarem atividades de formação coletivas para os trabalhadores do GHC. A maioria dessas atividades foi ofertada para o segmento assistência/ fim, o qual, efetivamente, representa o maior número de trabalhadores do GHC. Já as atividades realizadas pela instituição apresentavam uma característica diferente, pois, mesmo que tenham sido ofertadas, significativamente, para $\mathrm{o}$ segmento assistência/fim, também contemplou o segmento apoio/meio, que obteve quantitativo significativo de participação nas atividades. Mesmo não constando nos dados, na vivência cotidiana, pode-se observar que as atividades institucionais caracterizaram-se por uma tentativa de oferta de atividades multiprofissionais, e não somente de educação continuada por categorias profissionais, como se percebe nas atividades formativas realizadas pelas equipes.

Em relação às categorias profissionais, aglutinou-se algumas categorias por aproximação de processos, porém, constatou-se que, de maneira geral, as categorias assistenciais, como enfermeiros, médicos e técnicos e auxiliares de enfermagem, foram as categorias mais beneficiadas com as atividades de formação em 2013.

Quando se analisam as atividades de formação pela sua característica ou pelo tipo de formação, o resultado obtido é que as atividades mais corriqueiras são os "treinamento ou capacitações", os "cursos" e as "aulas ou palestras". Todas essas atividades são caracterizadas pela transmissão de conhecimento, onde o facilitador detém o conhecimento e o transmite aos ouvintes sem muito espaço para o questionamento e a troca de conhecimento.

Foi possível observar, através dos resultadosqueclassificaramastecnologiasdo cuidado em saúde, o grande contingente de atividades tanto realizadas pela instituição quanto pelas equipes aproxima-se mais das tecnologias leve/dura, seguidas pelas 
tecnologias duras e, por fim, pelas leves.

Esses resultados explicitam, em grande medida, o quanto à instituição ainda é permeada pelo modelo médico-hegemônico, o que repercute, diretamente, nas práticas educativas. Observa-se um conflito de identidade interno e a multiplicidade de modelos tecnoassistencias interagindo num mesmo espaço, uma vez que estes se encontram em disputa na instituição. O que resulta na presença de formações que têm como foco as tecnologias leves e os processos de trabalho vivo, apesar de, majoritariamente, a formação estar direcionada para a capacitação para uso de equipamentos, realização de procedimentos e conhecimentos estruturados, característicos do trabalho morto.

Este estudo traz resultados que podem contribuir na discussão das políticas de formação do GHC, porém novos estudos são fundamentais para corroborar com estes resultados.

\section{Referências}

${ }^{1}$ Yin RK. Estudo de caso: planejamento e métodos. Porto Alegre: Bookman; 2005. 3ed.

${ }^{2}$ Brasil. Ministério da Saúde. Grupo Hospitalar Conceição. Gerência de Informática. Sistema de informações administrativas. Porto Alegre: Grupo Hospitalar Conceição, 2014. Software.

${ }^{3}$ Brasil. Assembleia Constituinte. Constituição da República Federativa do Brasil. Brasília, DF: Senado, 1988. Disponível em:<http://www.senado.gov.br/sf/legislação/const/>. Acesso em: 09 jun 2014.

${ }^{4}$ Brasil. Ministério da Saúde. Grupo Hospitalar Conceição. Diretrizes organizacionais do GHC. Disponível em: <http://www.ghc.com.br/default.asp?idMenu=institucional\&idSubMenu=3>. Acesso em: 12 dez 2014.

${ }^{5}$ Brasil. Ministério da Saúde. Grupo Hospitalar Conceição. Escola GHC. Formação no GHC. Disponível em: <http://escola.ghc.com.br/index.php/escolaghc/formacao-no-ghc> Acesso em: 12 dez 2014

Ministério da Saúde, 2009.

Política Nacional de Educação Permanente em Saúde. Brasília:

${ }^{7}$ Brasil. Ministério da Saúde. Política Nacional de Gestão Estratégica e Participativa no SUS ParticipaSUS. Brasília: Ministério da Saúde, 2009.

${ }^{8}$ Brasil. Ministério da Saúde. Secretaria de Gestão do Trabalho Educação e da Educação na Saúde. Departamento de Gestão da Educação na Saúde. A educação permanente entra na roda: pólos de educação permanente em saúde: conceitos e caminho a percorrer. Brasília: Ministério da Saúde, 2005.

Documentos preparatórios para a 3을 Conferência Nacional de Gestão do Trabalho e da Educação na Saúde: trabalhadores da saúde e a saúde de todos os brasileiros práticas de trabalho, gestão, formação e participação. Brasília: Ministérios da Saúde, 2006. 
10 . Programa de Qualificação e Estruturação da Gestão do Trabalho e da Educação no SUS - ProgeSUS. Brasília: Ministério da Saúde, 2006.

${ }^{11}$ Merhy EE. O desafio que a educação permanente tem em si: a pedagogia da implicação. Interface - Comunicação, Saúde, Educação, Botucatu, 9(16): 172-174; 2005.

${ }^{12}$ Ceccim RB, Ferla AA. Educação e saúde: ensino e cidadania como travessia de fronteiras. Trabalho Educação e Saúde, Rio de Janeiro, 6(3): 443-456; nov. 2008/fev. 2009.

${ }^{13}$ Ceccim RB, Feuerwerker LCM. O Quadrilátero da formação para a área da saúde: ensino, gestão, controle social. Physis, Rio de Janeiro, 14(1): 41-65; 2004.

14 . Saúde: A cartografia do trabalho vivo. São Paulo. Hucitec, 2002.

${ }^{15}$ FRANCO, Túlio Batista; MAGALHÃES JÚNIOR, Helvécio Miranda. Integralidade na assistência à saúde. In: MERHY, Emerson Elias et al. (Orgs.) O Trabalho em saúde: olhando e experienciando o SUS no cotidiano. São Paulo: Hucitec, 2004. 2ed.

16 . Em busca do tempo perdido: A micropolítica do trabalho vivo em saúde. In: Merhy EE, Onocko R. (Org.). Agir em Saúde: um desafio para o público. São Paulo: Hucitec, 1997. p. 2-18.

${ }^{17}$ Franco TB. Healthcare Production and Pedagogical Out Put: Integration Of Healthcare System Settings In Brazil. Interface, Comunic., Saúde, Educ., 11(23): 427-438; set/dez 2007.

18

Reestruturação produtiva e transição tecnológica na saúde: um olhar a partir do cartão nacional de saúde. 2003. 19 f. Tese. (Doutorado em Saúde Coletiva - Universidade Estadual de Campinas). Campinas, 2003.

${ }^{19}$ Brasil. Ministério da Saúde. Grupo Hospitalar Conceição. Gerência de Recursos Humanos. Normas Regulamentadoras para Atividade de Formação - 441/2009 (NAF/GTED/GRH). Porto Alegre: Grupo Hospitalar Conceição, 2009.

${ }^{20}$ Brasil. Ministério da Saúde. Grupo Hospitalar Conceição. Gerência de Recursos Humanos. Dados dos Resultados de Formações Coletivas Realizadas em 2013. Núcleo de Atividades de Formação (NAF/GTED/GRH). Porto Alegre: Grupo Hospitalar Conceição, 2013. 